\title{
Separation of instant and accumulated nonlinear optical responses of dye-doped liquid crystal using Z-scan traces
}

\author{
Gayvoronsky V. ${ }^{1}$, Garashchenko V. ${ }^{1}$, Kislenko V. ${ }^{2}$, \\ Nikolaienko Yu. ${ }^{2}$ and Yakunin S. ${ }^{1}$ \\ ${ }^{1}$ Institute of Physics of NASU, 46 Nauka Ave., 03028 Kiev, Ukraine, \\ e-mail: vlad@iop.kiev.ua \\ ${ }^{2}$ Radiophysical Department, National Taras Shevchenko University, \\ 5 Academician Glushkov Ave., Kiev 01022, Ukraine
}

Received: 07.03.2007

After revision: 12.04.2007

\begin{abstract}
A theoretical approach is developed for distinguishing between the instant nonlinear optical response and the photoinduced accumulation effect on the refractive index. This is performed in terms of superposition of different lens transparencies for the known Z-scan technique. For the case of homeotropic liquid 5CB crystal cell doped with anthraquinone dye, we prove a possibility for decomposing consequent irreversible Z-scan traces into the contribution of instant nonlinear optical response, which takes place before the reorientation threshold, and the accumulated photoinduced refractive index variations occurred due to the exposition.
\end{abstract}

Keywords: liquid crystal, self-focusing, nonlinear optical effect, Gaussian beam, refractive index variation, Z-scan technique

PACS: 61.30.-v, 42.65.-k, 42.70.Df

\section{Introduction}

Investigation of nonlinear optical (NLO) characteristics of materials usually becomes complicated if different linear optical and NLO effects are simultaneously present. For transparent homogeneous materials, investigations of photoinduced changes due to selfaction of laser irradiation are usually performed, using the analysis of spatial beam profile in the far field. There are several widespread modifications of this method differing in the means employed for varying the beam intensity. These might be a variation of beam power at the optical system entrance (a so-called SPA technique - see [1]) or a variation of beam spot size on a sample. In the latter case the sample is usually moved through the beam waist, comprising the essence of a so-called Z-scan technique [2]. In spite of a series of advantages, the Z-scan method has some drawbacks. Variations of the spot size are objectionable for heterogeneous nanostructured materials. High levels of laser irradiation exposing samples in the vicinity of beam waist can ignite some irreversible 
photoinduced processes in the material under study, which have typically cumulative nature. As a result, a lot of novel optical functional materials exhibit complex NLO properties due to a joint impact of different photoinduced responses. In terms of on-axis Z-scan traces, the curves obtained experimentally for such the materials are deteriorated, when compare with typical butterfly-wing shape characteristics.

The idea of the present study is to develop a proper data treatment approach for the Z-scan experiments that use CW laser sources. It implies decomposing the mentioned complex response into an instant contribution (typically a cubic NLO response proportional to the actual laser field intensity inside the sample) and a cumulative one (proportional to the energy fluence). Our theoretical approach is tested on extremely efficient NLO material, which has been comprehensively studied, a nematic liquid crystal (LC) of 5CB doped with anthraquinone (AQ) dye.

The instant orientational response of the above LC doped with the AQ dye has been earlier investigated in $[1,3,4]$. The authors [1] have studied the NLO properties of the dye-doped LC using the techniques for registration of both the total transmitted power and the on-axis power in the far field. The NLO response of the dye-doped LC has revealed a large (one order of magnitude) difference for the cubic susceptibilities measured below and above the light-induced Freedericksz transition. The effect of the nonlinear refractive index sign inversion and the optical hysteresis properties for different light polarizations with respect to the LC director have been studied in the work [3]. Irreversible behaviour of the response has also been observed in the Z-scan traces after excitation by a train of femtosecond laser pulses [4]. The obtained data indicate a complicated response mechanism. Therefore it would be important to check the response of the nonlinear system as for the presence of dose effects and to develop the Z-scan technique $[2,5]$ so that to enable separating the contributions of different physical mechanisms. It has been already shown that the analysis of the results of Z-scan within the paraxial optics approach [5] should permit not only investigating a conventional selffocusing (or self-defocusing) but observing simultaneously accumulated photoinduced effects [5] that depend on the exposition time.

\section{Experimental}

In this work, nematic LC of 4'-n-pentyl-4-cyanobiphenyl (or simply 5CB) doped with 0.8 weight $\%$ of $N, N^{\prime}$-(4-methylphenyl)-1,4-diamino-AQ was used. Spectral dependences of its optical absorption and photoluminescence have been studied in [6]. Our experiments are performed for the cells with homeotropic LC alignment. The orientation has been induced by sputtering coating layers on the both glass substrates using $\mathrm{NiO}$ cathode [7]. The thickness of the LC layer has been set to be $100 \mu \mathrm{m}$.

The NLO experiments were carried out with a single-mode $\left(\mathrm{TEM}_{00}\right) \mathrm{CW} \mathrm{He}-\mathrm{Ne}$ laser (the wavelength $\lambda=633 \mathrm{~nm}$ and the power $16 \mathrm{~mW}$ ). Spatial intensity profile of the Gaussian beam was controlled with a CCD array AMKO LTI MuLTIray. The NLO properties were characterized while measuring the distortions of the focused laser beam 
passing through the sample under the normal incidence condition and the changes in the sample transmittance as a function of laser intensity and position of the sample (see Fig. 1). The focal length of the lens was equal to $8.5 \mathrm{~cm}$. The beam power at the entrance of optical system varied in the range of $1-70 \%$ with the aid of neutral attenuator having a transparency gradient.

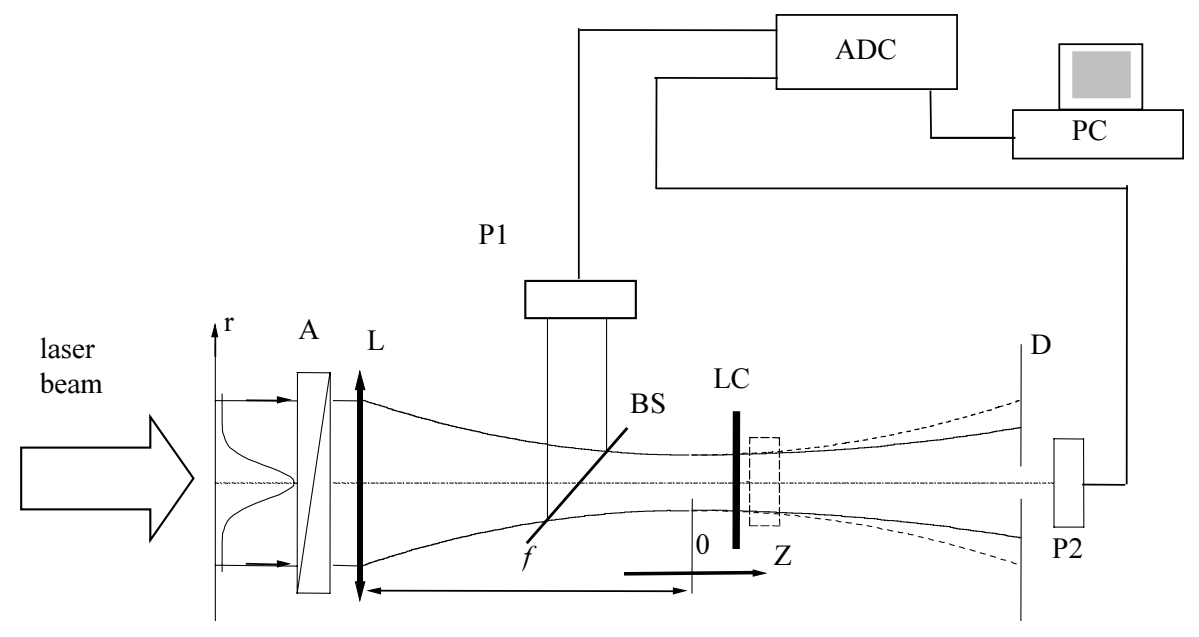

Fig. 1. Experimental setup: A - attenuator, BS - beam splitters, D - diaphragm for on-axis measurements, $\mathrm{L}$ - focusing lens with the focal length $f=8.5 \mathrm{~cm}$, LC - sample, P1 and P2 - photodiodes, and ADC - analog-to-digital converter.

The experimental setup contains two photodiode channels. The first one measures the laser beam power at the entrance of the optical system (the photodiode of this reference channel is indicated as P1 in Fig. 1). The second channel that employs the photodiode P2 measures the power passed through the sample in different geometries. As a result, the first channel deals with registration of the total transmitted power when the sample is positioned within the photodiode aperture. The second one is related to the onaxis power in the far field and so the photodiode $\mathrm{P} 2$ collects the on-axis part of the beam passed through a finite aperture diaphragm $\mathrm{D}(\varnothing=2 \mathrm{~mm}$ and the distance from the lens being $60 \mathrm{~cm}$ - see Fig. 1).

\section{Theory}

Let the film of a transparent or linearly absorbing material under analysis be confined by the planes $z=0$ and $z=l_{0}$, where $z$ axis corresponds to the optical axis of Z-scan setup (see Fig. 1). Notice that the problem is axially symmetric with respect to the optical axis of system, with $r$ being the distance from the axis to the point of observation. Let us consider deviation $\Delta n$ of the refraction index $n$ of our material from its statistically averaged value $n_{0}(\lambda)$, which occurs as a result of scan irradiation (the wavelength $\lambda$ and the electric field density $E(r))$. In addition to a common self-focusing contribution 
$\Delta n_{2}=n_{2}|E(r)|^{2}$, let us also account for the refraction index variation $\Delta n_{0}$ caused by the influence of various expositional effects, which depend on the power density $W(r)$ and the exposition time $t_{e}$ of the irradiation dose. In this work we restrict ourselves to consideration of only linear expositional effects, so that we have $\Delta n_{0}=\alpha \int_{0}^{t_{e}} W(t) d t$, where $\alpha=$ const.

Within our approach, we decompose the refractive index of the material into the finite series including a linear term and photoinduced variations:

$$
n=n_{0}+\Delta n_{0}\left(\lambda, W, r, t_{e}\right)+\Delta n_{1}\left(\lambda, r, I, t_{s}\right)+\Delta n_{2}(\lambda, E, r, z) .
$$

Here the term $\Delta n_{0}=\alpha \int_{0}^{t_{e}} W(t) d t$ is responsible for the refractive index changes caused by a prior irradiation of sample by the quanta of arbitrary energy, with the transversal distribution of power density $W(r, t)$ and the exposition time $t_{e}$. The term $\Delta n_{1}=\beta \int_{0}^{t_{s}} I(t) d t$ describes a cumulative photoinduced variation of the refractive index proportional to the energy fluence of the laser radiation during the current Z-scan trace, with a constant coefficient $\beta=$ const. Finally, the third term $\Delta n_{2}=n_{2}|E(r)|^{2}=\gamma I$ describes an instant NLO response corresponding to the self-focusing (or self-defocusing) effect of the laser beam within the sample $(\gamma=$ const $)$. Below we will demonstrate that all these factors could be correctly considered and analyzed with the methods of paraxial optics $[5,8]$.

In case of $\Delta n_{2}>\Delta n_{0,1}$, the refractive index variation in Eq. (1) may be approximately expressed in terms of the average expositional doses $\bar{W}=\frac{1}{t_{e}} \int_{0}^{t_{e}} W(t) d t$ and $\bar{I}=\frac{1}{t_{e}} \int_{0}^{t_{s}} I(t) d t$

$$
\Delta n=\Delta n_{0}+\Delta n_{1}+\Delta n_{2},
$$

where $\Delta n_{0} \approx \alpha \bar{W} t_{e}, \Delta n_{1} \approx \beta \bar{I} t_{s}$ and $\Delta n_{2}=\gamma I$.

Let us now concentrate on the lens transparency (or the normalized transmittance) of the Z-scan scheme. The Gaussian beam used in frame of the Z-scan technique has axially symmetric distribution of the amplitude of electric field over the cross section $(z=$ const $)$ :

$$
E(z, r)=E_{0}(z) \exp \left(-r^{2} / 2 \rho_{2}^{2}(z)\right),
$$


where $E_{0}(z)$ is the on-axis electric field amplitude of the laser beam and $\rho_{2}(z)$ the beam radius [9].

Propagation of a Gaussian beam through a thin material layer of the thickness $l_{0}$ with axially variable refractive index is equivalent to its propagation through the same material layer, but with a constant refractive index $n_{0}$ and an effective thickness $l_{\text {eff }}$. The latter is determined from the condition of equality of the optical path lengths in the both cases [5]:

$$
l_{\text {eff }} n_{0}=l_{0} n
$$

Taking Eqs. (1) and (4) into account, one readily obtains the deviation of $l_{\text {eff }}$ from the geometrical layer thickness $l_{0}$ :

$$
\Delta=l_{\text {eff }}-l_{0}=\Delta n l_{0} / n_{0} .
$$

Let the axially symmetric distributions of the parameters $\Delta n_{i}$ have a Gaussian profile:

$$
\Delta n_{i}(r)=\Delta n_{i}(0) \exp \left(-r^{2} / \rho_{i}^{2}\right),
$$

where $\Delta n_{i}(0)$ are the amplitude values on the optical axis of the system $(r=0)$ and $\rho_{i}$ the corresponding radiuses.

Within the Z-scan scheme, one uses only on-axis paraxial rays, which is equivalent to paraxial approximation of the geometrical optics. The complex wave surface for this paraxial region $(r \approx 0)$ can be replaced by a tangent sphere. Accounting for Eq. (6), one can determine the radius $R_{\Sigma}$ of the latter as [10]

$$
R_{\Sigma}=\left[1+(\partial \Delta / \partial r)^{2}\right]^{3 / 2} /\left(\partial^{2} \Delta / \partial r^{2}\right)_{r=0}=n_{0} / \sum_{i=0}^{2}\left(2 l_{0} \Delta n_{i}(0) / \rho_{i}^{2}\right) .
$$

The sign in Eq. (7) is chosen from the condition that the domain where $\Delta n>0$ would correspond to the value $R_{\Sigma}>0$.

In frame of the paraxial approximation, the material layer under analysis acts as a set of thin spherical lenses, with the total focal power at the exit surface [11] defined by

$$
\Phi_{\Sigma}=\left(n_{0}-1\right) / R_{\Sigma}=\sum_{i=0}^{2} \Phi_{i},
$$

with $\Phi_{i}=\frac{\left[2 l_{0} \Delta n_{i}\left(n_{0}-1\right)\right]}{\left(n_{0} \rho_{i}^{2}\right)}$. In our case the term $\Phi_{0}=\frac{\left[2 l_{0} \alpha W(0) t_{e}\left(n_{0}-1\right)\right]}{\left(n_{0} \rho_{0}{ }^{2}\right)}$ means a focal power of the photoinduced lens recorded due to the accumulation effect during previous traces (i.e., the initial focal power for the current trace), $\Phi_{1}=\frac{\left[2 l_{0} \Delta n_{1}\left(\lambda, 0, t_{s}\right)\left(n_{0}-1\right)\right]}{\left(n_{0} \rho_{1}^{2}(z)\right)}$ is a 
cumulative focal power of the analyzed layer acquired during the current scan period $t_{s}$ (it is proportional to the energy fluence, i.e., $\left.\Phi_{1} \sim \int_{0}^{t_{s}} I(t) d t\right)$ and, finally, the term $\Phi_{2}=\frac{\left[2 l_{0} n_{2}|E(0)|^{2}\left(n_{0}-1\right)\right]}{\left(n_{0} \rho_{2}^{2}(z)\right)}$ represents a focal power of the instant NLO lens due to the self-focusing or self-defocusing effects.

The function $\rho_{2}(z)$ can be defined either through the radius $\rho_{2}(-F)$ of the input beam in the plane of the focusing lens or, alternatively, through the minimum radius $\rho_{2}(0)$ of the beam in the region of focal waist. These relations are specified by the wellknown expressions [9]

$$
\rho_{2}^{2}(z)=\left(\rho_{2}(-F) z / F\right)^{2}+\left[(z+F) k \rho_{2}(-F)\right]^{2},
$$

or

$$
\rho_{2}^{2}(z)=\rho_{2}{ }^{2}(0)\left(1+z^{2} / z_{d}^{2}\right),
$$

where $k=2 \pi / \lambda$ is the wave number, $\lambda$ the wavelength (notice that the both quantities are determined for a free space) and $z_{d}=k \rho_{2}{ }^{2}(0) / 2$ the diffraction length of the beam. Analogous expressions may also be written for the parameter $\rho_{1}(z)$.

The lens transparency (or the normalized transmittance) $\tau(z)$ for the Z-scan technique could be defined as a reciprocal of the square of relative focal power of the sample [5]. Its approximate form is as follows:

$$
\tau(z)=1+2 z \Phi_{\Sigma}=1+2 z\left(\Phi_{0}+\Phi_{1}+\Phi_{2}\right) .
$$

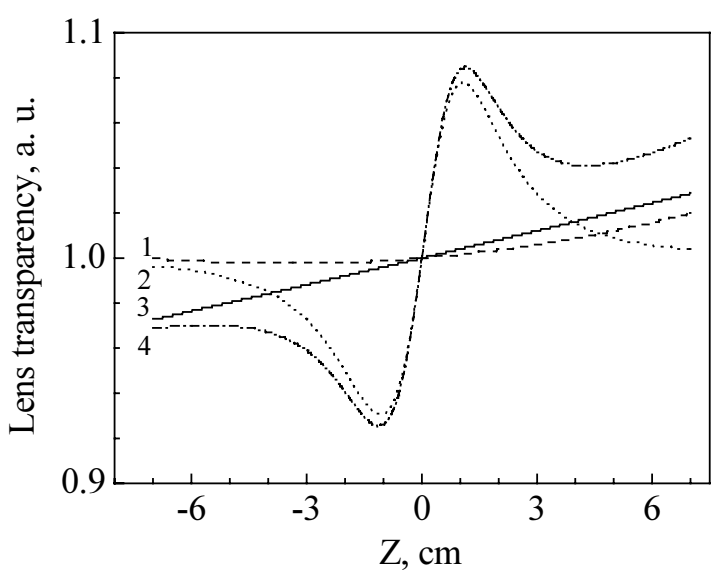

Fig. 2. Dependences of the lens transparency $\tau(z)$ of NLO medium for different mechanisms of $\Delta n_{i}$ variations. Curve 1 corresponds to accumulated contribution caused by irradiation during a current Z-scan trace, curve 2 describes a direct self-focusing effect, curve 3 is the initial lens transparency of the medium imposed in the layer by exposition effects and curve 4 shows a superposition of all the mentioned effects. 
The examples of numerical simulation of the lens transparency for different specific mechanisms of $\Delta n_{i}$ changes $(i=0,1,2$ and $\Sigma)$ are shown in Fig. 2.

Curve 1 in Fig. 2 corresponds to contribution of the cumulative response, which is described in terms of the photoinduced lens with the focal power $\Phi_{1}$ proportional to the laser beam energy fluence. Curve 2 describes the instant response due to self-focusing effect, where the focal power of the self-focusing lens is equal to $\Phi_{2}$. Curve 3 in Fig. 2 gives the influence of the initial lens, with the focal power $\Phi_{0}$ recorded in the layer before the current Z-scan trace illumination. Finally, the dependence of the lens transparency represented by curve 4 shows the total of all the effects mentioned above.

\section{Results and discussion}

As seen from Fig. 3, homeotropic LC cell reveals a self-focusing property indicated by increasing on-axis transmittance versus the laser intensity occurred in the geometry when the sample is located behind the beam waist. The effect is also evident from the form of the Z-scan trace of the on-axis transmittance (see Fig. 4).
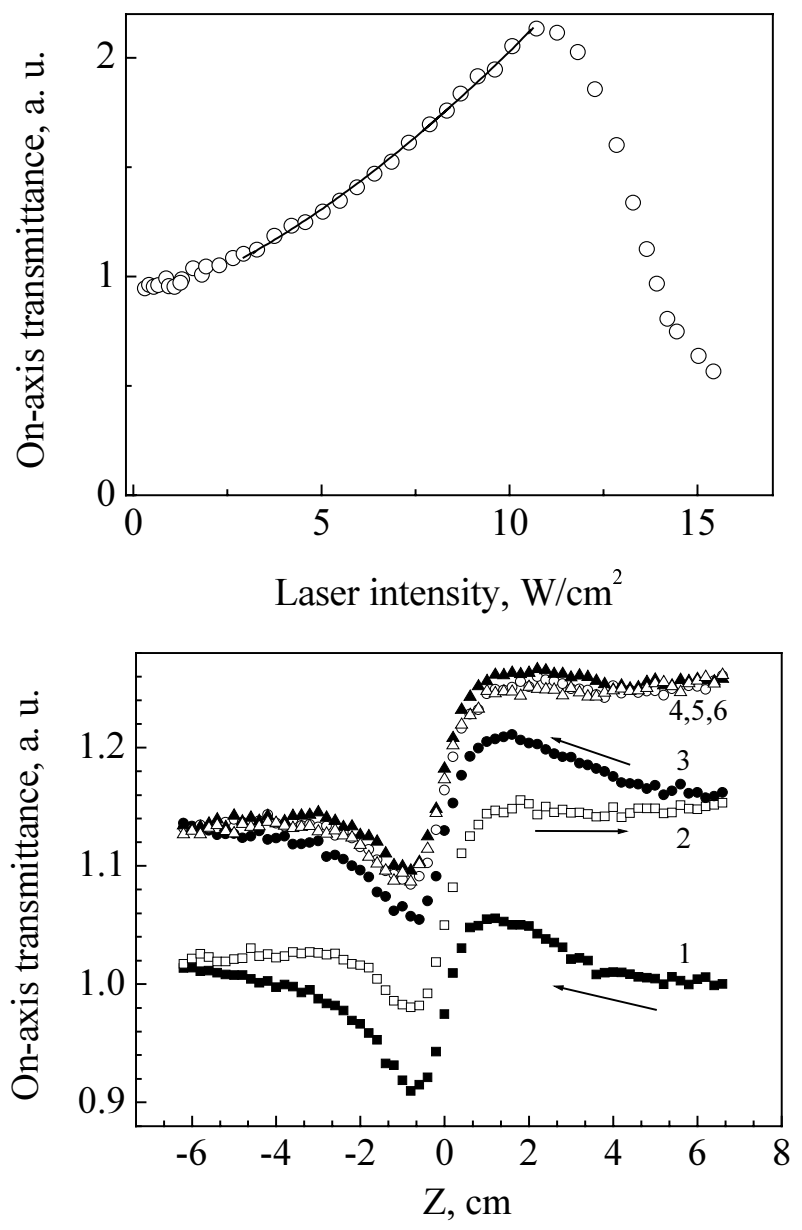

Fig. 3. On-axis transmittance vs. laser intensity: circles are the experimental data and line corresponds to approximation within the model mentioned in the text.

Fig. 4. Experimental data obtained for the six successive Z-scans of the same region. Full symbols correspond to the forward traces $(1-\mathbf{m}, 3-\bullet$ and $5-\boldsymbol{\Delta}$ ) and open ones to the backward traces $(2-\square, 4-\circ$ and $6-\Delta)$. 
The dependence of the on-axis transmittance upon the laser intensity has a monotonic, nearly linear behaviour in the range of initial intensities. When the intensity exceeds the level of $10 \mathrm{~W} / \mathrm{cm}^{2}$, the on-axis transmittance drops down sharply. As shown in $[3,4]$, the forward and backward intensity changes for the on-axis transmittance after reorienting LC manifest a hysteretic behaviour. However the changes taking place in the overall range of initial intensities are reversible whenever the exposure time is about several minutes. For this case we have been able to fit the experimental on-axis transmittance data with a single reversible photoinduced lens $\Phi_{2}$ and to estimate the corresponding parameter $\gamma$. The fitting performed within the model described in [1] is presented in Fig. 3 as a solid line.

The dose effects are observed in the Z-scan experiments when the exposure time is approximately equal to half an hour. Then the laser beam power has been set to the value corresponding to the beam waist intensity typical for the nearly-linear monotonic behaviour of the on-axis transmittance $\left(I \sim 6 \mathrm{~W} / \mathrm{cm}^{2}-\right.$ see Fig 3$)$. The results of the closed-aperture Z-scan are presented in Fig 4, where full symbols correspond to the forward traces (curves 1, 3 and 5) and open ones - to the backward traces (curves 2, 4 and 6). We mark that the traces are no longer reversible. The curve edges are not located on the same transmittance level and each next trace is moved upward. The accumulation changes saturate at the fourth trace, afterwards all the next traces coincide with each other.

In order to exclude the effects of nonlinear absorption, all of the obtained experimental curves (Fig. 4) have been normalized by means of dividing the resulting transmittance by the lens transparency $\tau(z)$ obtained with the opened aperture (see Fig. 5). The dependences of total transmittance show a weak effect of photoinduced darkening in the beam waist region (about $3 \%$ of the linear optical transmittance of the sample). The total Z-scan transmittance traces are symmetric and independent of the number of traces. The total transmittance traces correspond to the intensity dependences obtained with the SPA technique. This indicates that the accumulation response is caused by the refractive index variation only, without appreciable variations of the absorption.

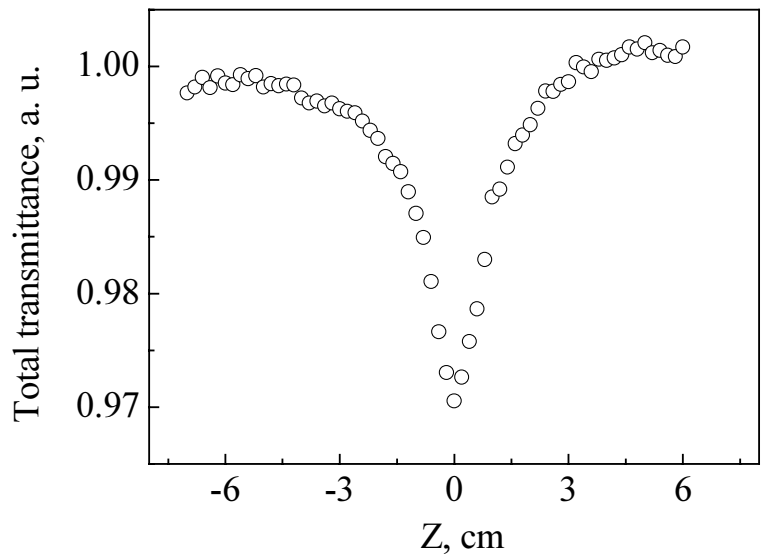

Fig. 5. Results of Z-scan with the open aperture. 
To study the irreversible expositional optical effects, we have carried out repeated Z-scans of the sample in the same region. Fig. 4 reflects the experimental results of the Z-scan for the first (curve 1), third (curve 2) and eighth (curve 3) measuring cycles.

\section{Analysis and discussion of the results}

The experimental on-axis transmittances and the dependences of the lens transparency $\tau(\mathrm{z})$ computed according to Eq. (11) for the first, the third and the sixth Z-scan trace (dependences 1,2,3 accordingly) are compared in the Fig. 6The lens transparency has been determined for the case of $\Delta n \approx \beta \bar{I} t_{s}+\gamma I$, where $\bar{I} \approx 1 / t_{s} \int I(t) d t$ denotes the effective intensity of the current scan.

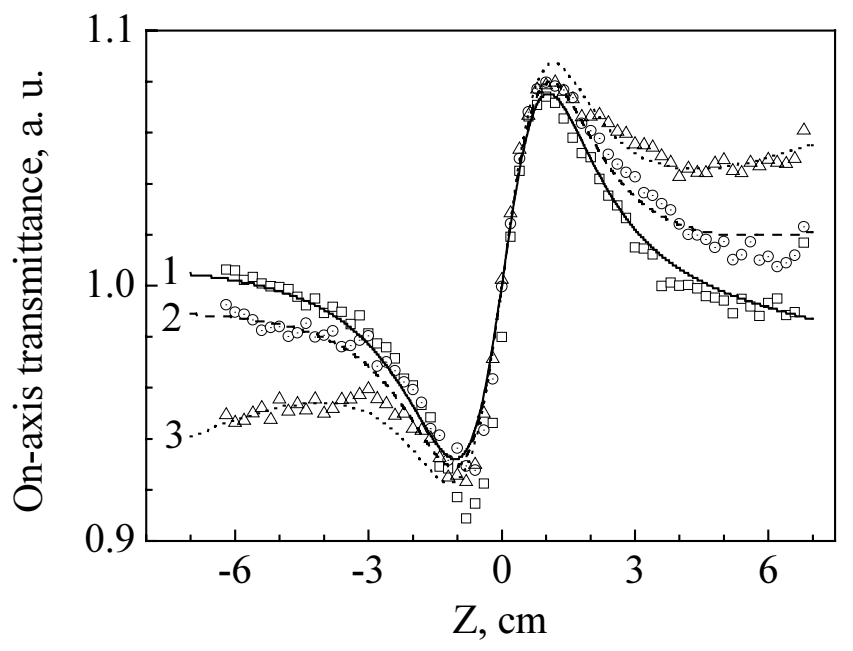

Fig. 6. Experimental data and theoretical Z-scan traces of on-axis transmittance for the first, the third and the sixth Zscan trace (dependences $1,2,3$ accordingly)

Using the technique suggested above, we have thus analyzed the three following kinds of contributions to the photoinduced refractive index changes: the instant response, the cumulative response during a single scan and the impact of the changes (recorded lens) during the previous scans. We have determined a number of NLO parameters for the sample of nematic LC 5CB doped with $0.8 \%$ AQ dye. Namely, we have obtained the basic parameters $\gamma=315 \mathrm{~cm}^{2} / \mathrm{MW}$ and $\beta=8.9 \cdot 10^{-4} \mathrm{~cm}^{2} / \mathrm{MJ}$. Notice that estimations of the instant NLO coefficient for the refractive index made on the basis of SPA technique data (see Fig. 3) gives a close value $\gamma=270 \mathrm{~cm}^{2} / \mathrm{MW}$. In other words, the magnitudes of the instant response obtained using the Z-scan and the SPA techniques coincide with the accuracy of about $15 \%$, which corresponds to the measurement errors.

\section{Conclusions}

The experimental and theoretical results of the present work testify that the sample of nematic LC, 5CB doped with $0.8 \%$ AQ dye, manifests simultaneous expositional (accumulative) and self-focusing effects upon the radiation of He-Ne laser with the wavelength $\lambda=633 \mathrm{~nm}$. 


\section{Acknowledgements}

This work has been supported by the NASU grants V-114 and VC-139. We acknowledge R. Vasyuta for the preparation of the samples and V. Nazarenko for helpful discussion.

\section{References}

1. Gayvoronsky V, Yakunin S, Nazarenko V, Starkov V and Brodyn M, 2005. Techniques to Characterize the Nonlinear Optical Response of Doped Nematic Liquid Crystals. Mol. Cryst. Liq. Cryst. 426: 231-241.

2. Sheik-Bahae M, Said AA, Wei TH, Hagan DJ and Van Stryland EW, 1990. Sensitive measurement of optical nonlinearities using a single beam. IEEE J. Quant. Electron. QE 26: 760-769.

3. Gayvoronsky VYa, Yakunin SV, Pergamenshchik VM and Nazarenko VG, 2006. Sign inversion of the optical torque on the nematic director enhanced by anthraquinone dye dopants stable to the light action. Laser Phys. Lett. 3: 531-535.

4. Gayvoronsky V, Yakunin S, Enikeeva V and Ozheredov I, 2006. Selfaction effects of femtosecond laser pulses in dye-doped 5CB liquid crystal. Laser Phys. Lett. 3: 357361.

5. Kislenko VI, Stetsyuk VN and Maevskaya OV, 2006. Determination of the lens transmittance of nonabsorbing media using the optical matrix method. Optics and Spectroscopy. 100: 631-635.

6. Gayvoronsky V, Yakunin S, Pergamenshchik V, Nazarenko V, Palewska K, Sworakowski J, Podhorodecki A and Misiewicz J, 2006. Photoluminescence of Nematic Liquid Crystal Doped with Anthraquinone Dye. Ukr. J. Phys. Opt. 7: 116123.

7. Cognard J. Alignment of Nematic Liquid Crystals and Their Mixtures. London: Gordon and Breach (1982).

8. Kislenko V.I. and Nikolaenko Yu.N. Z-scan investigation of the optical exposition effects. Proc. of the Sixth International Young Scientist's Conference on Applied Physics, Kyiv, Ukraine, (2006) p.26.

9. Akhmanov S.A. and Nikitin S.Yu. Physical Optics. New York: Oxford University Press (1997).

10. Korn A. and Korn M. Curvature of a Plain Curve. In Mathematical Handbook for Scientists and Engineers. New York: McGraw-Hill (1968), p. 499.

11. Gerrard A. and Burch J.M., Introduction to Matrix Methods in Optics. New York: Dover (1994). 